\title{
PAUTAS PARA EVALUAR EL DESEMPEÑO DE UNA UNION ADUANERA, CON ESPECIAL REFERENCIA AL COMERCIO INTRAINDUSTRIAL. EL CASO DEL MERCOSUR*
}

\section{Introducción}

Este trabajo se propone analizar los efectos previstos de una unión aduanera en formación -el MERCOSUR-respecto a ciertas actividades industriales y en el contexto de globalización y regionalización imperante a nivel mundial. En tal sentido, el objetivo principal lo constituye la evaluación de los flujos comerciales entre los países miembro del MERCOSUR y según sectores de la actividad industrial; a tal fin se analizan tanto aspectos tradicionales -creación y desvío de comercio-asi como otros relativamente novedosos en la literatura económica, v.g. el comercio intraindustrial, procurando la contrastación empírica de ciertas hipótesis de la teoría económica.

En la primera sección se explicitan los fundamentos teóricos de una unión aduanera, así como también del llamado "comercio intraindustrial", suministrándose diversos indicadores para la cuantificación de este último y de los efectos creación y desvío de comercio. Finalmente, se enuncian criterios alternativos para evaluar el desempeño de una unión aduanera.

La segunda sección expone los resultados empíricos obtenidos acerca de los tres tópicos mencionados en el análisis teórico, i.e. creación y desvio de comercio; comercio intraindustrial y criterios alternativos.

El trabajo concluye con la presentación y discusión de los principales resultados obtenidos.

* Este trabajo constituye una reproducción parcial de la Tesis de Magister en Economía:"Condiciones y perspectivas de la integración industrial selectiva. El caso del Mercosur", presentada por la autora en el Departamento de Graduados de la Universidad Nacional del Sur, año 1994. 


\section{Fundamentos teóricos}

\section{I.1. Introducción a la teoría de la unión aduanera}

La teoría de la unión aduanera se ocupa de los efectos que las variaciones en las barreras arancelarias geográficamente discriminantes (1) ejercen sobre el bienestar económico. La decisión de concertar una zona de libre comercio o una unión aduanera abre camino a la realización de un proyecto destinado a eliminar los obstáculos político-comerciales, condicionantes de las relaciones económicas mutuas.

Una zona de libre comercio es aquella en la cual el intercambio de bienes entre los países miembro se libera progresivamente de todos los aranceles y cuotas, pero cada socio conserva simultáneamente completa autonomía en su politica comercial respecto a terceros paises. Este concepto es fácil de implementar desde el punto de vista político, dado que no restringe el margen de soberania de los países socios, i.e. cabe la posibilidad de mantener vigentes los convenios preferenciales existentes con terceros países. Ejemplos de zonas de libre comercio son EFTA (Asociación Europea de Libre Comercio) y ALALC (Asociación Latinoamericana de Libre Comercio), ambas fundadas en 1960 .

La unión aduanera reúne características similares a la zona de libre comercio, pero difiere de ésta en que se igualan progresivamente los aranceles y cuotas empleados individualmente por los países miembro con respecto a terceros países. Asimismo, se procura una política comercial común frente al resto del mundo. Ejemplos de unión aduanera son la Unión Aduanera Alemana (1834) y la de Benelux (1948), transformada en Comunidad Económica Europea desde 1958.

Etapas ulteriores en la integración económica son el mercado común, que a la caracterización de la unión aduanera agrega el movimiento irrestricto de factores de producción- mano de obra y capital- entre los países miembro y la unión económica, concebida como un mercado común al que se agrega el requisito de armonizar políticas fiscales, monetarias y socioeconómicas entre los miembros. 


\section{I.2. Criterios de creación y desvío del comercio}

El núcleo del análisis de la teoría ortodoxa de la unión aduanera se basa en la diferenciación y ponderación de los efectos creación y desvío del comercio, postulados por Viner en 1950 (2). Hasta ese momento se afirmaba que "siempre, o casi siempre, las uniones aduaneras aumentarán el bienestar" (3), puesto que se disminuyen los aranceles y, en consecuencia, se genera un movimiento hacia el libre cambio y el incremento del comercio mundial.

Viner demostró, no obstante, que el sesgo de una unión aduanera, es el resultado neto de dos fuerzas opuestas, i.e. creación y desvío de comercio.

La creación de comercio (CC) surge en tanto un país A que posee condiciones de producción en una industria más favorables que el país $\mathrm{B}, \mathrm{y}$ que antes producía sólo para el mercado doméstico, está ahora en condiciones de exportar al pais $\mathrm{B}$ debido a que los aranceles en disminución provocan precios más bajos de sus productos en mercados externos. Los factores de producción se desplazan hacia las producciones nacionales en las cuales cada pais tiene ventajas comparativas (efecto producción) y aumenta el consumo debido a los precios relativos más favorables (efecto consumo), con el consiguiente aumento del bienestar mundial y el de los miembros de la unión.

En el caso del desvío de comercio (DC), un productor más eficiente fuera de la unión aduanera es desplazado por otro productor con mayores costos perteneciente a un país socio, en virtud de las preferencias arancelarias que lo favorecen. Por lo tanto, el desvío de comercio sería económicamente indeseable. No obstante, cabe destacar que este concepto supone que los aranceles constituyen la barrera comercial prevaleciente. A medida que las barreras no-arancelarias se tornan características más destacadas del marco proteccionista, la conclusión no es tan evidente, ya que el socio que reemplaza a los terceros paises como fuente de suministro puede tener sus exportaciones limitadas por las restricciones cuantitativas de otro país socio y no por tener mayores costos.

Los efectos CC y DC pueden visualizarse a través del siguiente ejemplo (4):

Los países A, B y C establecen una unión aduanera. Los agentes económicos de los tres países miembro demandan el bien "X", el cual sólo se produce en $\mathrm{B}$ y en $\mathrm{C}$. Además, " $\mathrm{X}$ " se produce también en terceros países (D). 
Se supone que el oferente del bien " $\mathrm{X}$ " localizado en B fija un precio de 120 , aquel ubicado en $\mathrm{C}$ uno de 130 y en $\mathrm{D}$ uno de 110 . La tarifa de importación original (ad-valorem) corresponde en el caso A al 10\%; en B a $20 \%$ y en $\mathrm{C}$ a $30 \%$. La tarifa externa común, al establecerse la unión aduanera, podría ascender a $20 \%$. Antes de la unión aduanera, la economía $\mathrm{A}$, que no produce el bien "X", lo importa desde terceros países; los consumidores en B y $\mathrm{C}$ se abastecen prioritariamente de la producción nacional de " $\mathrm{X}$ " a causa de la política proteccionista.

\begin{tabular}{|c|c|c|c|c|c|c|c|c|}
\hline \multirow{2}{*}{$\begin{array}{l}\mathrm{P} \\
\mathrm{A} \\
\mathrm{I} \\
\mathrm{S}\end{array}$} & \multicolumn{8}{|c|}{$\begin{array}{l}\text { Precio en el mercado interno de las importaciones } \\
\text { del bien X desde : }\end{array}$} \\
\hline & \multicolumn{4}{|c|}{ Antes de la unión } & \multicolumn{4}{|c|}{ Después de la unión } \\
\hline A & . & 132 & 143 & 121 & & 120 & 130 & 132 \\
\hline B & . & 120 & 156 & 132 & & 120 & 130 & 132 \\
\hline C & . & 156 & 130 & 143 & & 120 & 130 & 132 \\
\hline
\end{tabular}

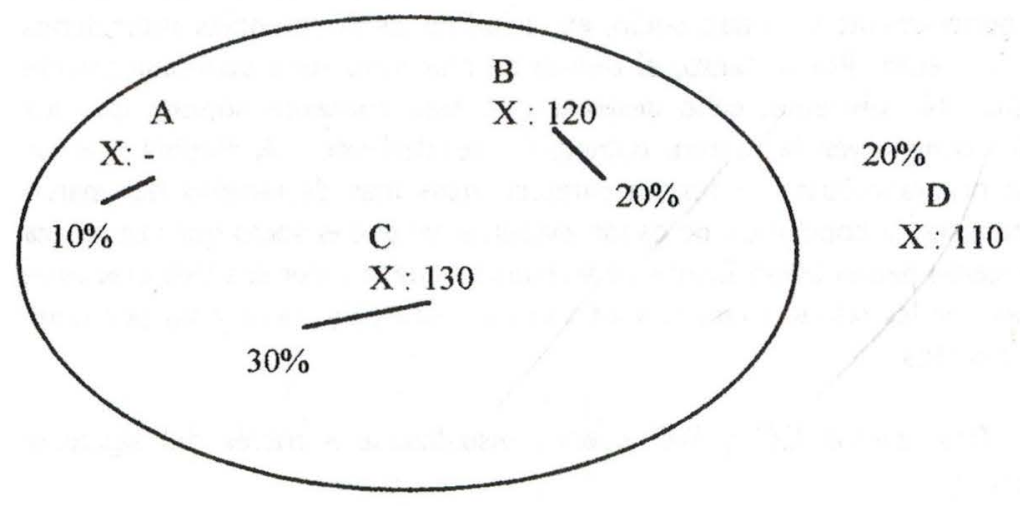

La realización de la unión aduanera tiene como consecuencia que los productores de $\mathrm{B}$, e incluso también aquellos de $\mathrm{C}$, podrán ahora ofrecer el 
bien " $\mathrm{X}$ " en el país A más barato que el productor mundial más favorecido en D. Ello significa que una fracción de la expansión del comercio entre los países miembro acontece exclusivamente a costo de la disminución de las importaciones desde el resto del mundo(DC). Asimismo, después de la formación de la unión aduanera, el pais B está en condiciones de autoabastecerse y abastecer al país $\mathrm{C}$ a un costo menor que antes, lo cual da lugar a una creación de comercio entre estos países, supuesto que se produce el desplazamiento de factores previsto en virtud de las localizaciones más eficientes.

\section{I.3. Criterios alternativos}

Si bien la diferenciación conceptual entre creación y desvío de comercio es clara, la cuantificación del saldo neto resultante de ambos efectos (potencial del proyecto integracionista) presenta serias dificultades. En consecuencia, el análisis económico se ha centrado en dos cuestiones: a) condiciones para la ocurrencia de los efectos creadores y desviadores del comercio y b) supuestos bajo los cuales estas condiciones determinan el aumento o disminución del bienestar económico mundial ante la formación de una unión aduanera. A tal efecto se han formalizado cinco criterios económicos (5):

\section{Monto y estructura arancelaria vigente previo a la formación de la unión aduanera}

En el caso de bienes importados desde paises miembro, la disminución de tarifas provoca una tendencia (relativa) a la disminución de precios. Las ventajas así resultantes debido a una elevada relación creación/desvio de comercio (i.e. mejora de los ingresos reales y rentas preferenciales para los productores ubicados dentro de la unión aduanera), pueden ser tanto mayores cuanto más elevados sean los aranceles anulados entre los países miembro y cuanto mayor sea la diferencia entre los aranceles aplicados a terceros países antes del establecimiento de la unión y el arancel externo común aplicado después de la instauración de aquella. El efecto neto depende no sólo del monto de los aranceles previos, sino también de la estructura original de los aranceles nacionales. 


\section{Monto de las cuotas inicialmente vigentes}

Si previo al establecimiento de una unión aduanera se aplican restricciones cuantitativas a la importación respecto a terceros países y éstas no se reducen en virtud de la unión aduanera, el efecto proteccionista elevado- de las cuotas no se acrecienta. En efecto, si el productor más eficiente del bien en cuestión residiera en un tercer país, puede seguir abasteciendo a los países en la misma medida que antes del establecimiento de la unión.

\section{Estructura productiva de los países miembro y del resto del mundo previo a la formación del bloque}

Cuanto más similar sea la estructura productiva de los paises miembro y, por lo tanto, mayor el grado de competencia mutua entre ellos como consecuencia de los aranceles vigentes originariamente (bienes sustitutivos), mayor será la presión resultante de la disminución de aranceles dentro de la comunidad para desplazar fuerzas productivas desde localizaciones comparativamente poco productivas hacia usos más eficientes.

Asimismo, si las economías de los países son semejantes, éstos seguirán importando desde terceros paises bienes no producidos por ellos y el desvio de comercio será reducido. En cambio, si la asociación se verifica entre economías cuyas ofertas de bienes son complementarias, las perspectivas de un aumento significativo de la productividad son insignificantes y la probabilidad de desvío respecto a terceros países es grande (medido en términos de recorte a las importaciones desde aquellos).

Inversamente, cuanto más complementaria es la oferta de bienes de terceros países con respecto a la producción de los socios en la unión aduanera, tanto más podrá beneficiarse el resto del mundo de la aceleración del crecimiento de aquella.

Una justificación alternativa en favor de los países miembro con economías complementarias está basada en sus estructuras de costos en relación con los costos de terceros países, i.e. la complementariedad es conveniente si los costos de producción de los paises miembro se aproximan (o se hallan por debajo) de los costos mundiales, resultando beneficios 
elevados por unidad de comercio creado y costos reducidos por unidad de comercio desviado.

\section{Interdependencia económica de los países miembro previo al establecimiento de la unión aduanera}

Una unión aduanera producirá un resultado neto ventajoso cuanto mayor sea la magnitud del comercio entre los países miembro, previo a la asociación. Las posibilidades para el surgimiento de desvio de comercio son tanto menores cuanto menor sea, al momento de crearse la unión, la participación de terceros países en las importaciones totales de los países miembro. La unión fortalecerá los patrones de intercambio natural y no los desviará de manera artificial.

\section{Potencial económico mundial de los países asociados}

El predominio de los efectos ventajosos de la unión aduanera será tanto más probable, i.e. menor desvío de comercio, cuanto mayor sea la participación en la producción y el consumo mundiales del comercio liberado de las restricciones existentes. La participación del intercambio intra-bloque en relación al respectivo volumen nacional de comercio exterior resulta, asimismo, tanto más elevada cuanto mayor sea el número de países que forman la unión aduanera. No obstante, a partir de un tamaño determinado, aparecen efectos negativos para el comercio con terceros países, i.e. la unión tiende a ser más autárquica.

\section{I.4. Comercio intraindustrial}

El comercio intraindustrial de un país -conocido como "comercio en dos sentidos"- está compuesto por exportaciones e importaciones simultáneas de productos pertenecientes a la misma categoría industrial, verificándose una especialización entre subsectores de una misma actividad económica. La identificación y medición del fenómeno depende en gran medida del grado y clase de homogeneidad de los bienes incluidos en cada grupo estadístico.

El comercio intraindustrial puede verificarse no sólo entre productos heterogéneos, sustitutos relativamente cercanos, sino también entre productos homogéneos, diferenciados por su disponibilidad temporal (ejemplo: energía, 
comercio fronterizo) $\mathrm{y}$, sobre todo, por sus costos de transporte, almacenamiento y comercialización, vg: productos agrarios.

A principio de los años ' 60 , algunos economistas se interesaron por la evolución de los patrones comerciales, resultantes de la formación de la Comunidad Europea. Los conocimientos convencionales sobre los efectos de la unión aduanera (6), sugerían que la especialización subsiguiente a la liberalización del comercio sería de tipo inter- industrial. Sin embargo, Dreze, Verdoorn y Balassa (7) encontraron evidencias de especialización intraindustrial creciente en la década siguiente a la formación de uniones aduaneras. Desde la publicación de Grubel y Lloyd (8) ha surgido una vasta literatura sobre el tema.

El fenómeno del comercio intraindustrial no está contemplado por la teoría clásica pura del comercio internacional, derivada de Ricardo, Heckscher y Ohlin. Los intentos por generalizar estos modelos no incorporan convenientemente el rol desempeñado por las economías de escala y la diferenciación del producto. En consecuencia, el postulado según el cual los paises se especializan y exportan los bienes que usan más intensamente el factor de producción relativamente abundante, fracasan al intentar explicar el comercio intraindustrial.

En virtud de ello, diversos autores (9) han ofrecido un nuevo tratamiento teórico del tema, destinado a suministrar un marco de análisis realista de los modernos flujos comerciales.

\section{Medición del comercio intraindustrial}

Existen varios criterios para medir la especialización intraindustrial $(10)$ :

\subsection{Coeficiente de correlación de rango (Spearman) (11)}

Relaciona las estructuras de exportación por pares de paises, i.e. dados dos países $\mathrm{A}$ y $\mathrm{B}$ permite medir qué relación puede haber entre las exportaciones del país A hacia - y sus importaciones desde- el país B (siendo estas últimas equivalentes a las exportaciones de $\mathrm{B}$ hacia $\mathrm{A}$ ). Las variables a considerar serian, para un año dado, el valor de las exportaciones de A hacia 
B y de B hacia A, ambos por secciones industriales, y los rangos correspondientes a dichos valores, dando el rango uno al valor más grande.

El coeficiente se formula como:

$$
\mathrm{r}=1-\left\{6 * \sum_{\mathrm{i}=1}^{\mathrm{n}} \mathrm{di}^{2} /\left[\mathrm{n} *\left(\mathrm{n}^{2}-1\right)\right]\right\}
$$

donde :

d: diferencia entre los rangos por pares de valores de comercio bilateral.

$\mathrm{n}$ : número de pares de observaciones.

El valor de $\mathrm{r}$ oscila entre $(-1)$ y $(+1)$. Si los rangos de los valores de exportación recíproca por industria concuerdan perfectamente, cada $\mathrm{di}=0 \mathrm{y}$ $r=1$. Si los rangos asignados a las exportaciones de un país son exactamente los opuestos a los de las exportaciones recíprocas del otro país, $\mathbf{r}=-1$. Si $r=0$, no existe relación alguna entre las dos estructuras de exportación.

Este índice fue empleado por Balassa en el análisis del comercio intra-CEE.

1.2. Relaciones representativas (12)

$$
x_{i}=\frac{|X i-M i|}{(X i+M i)}
$$

donde

$\mathrm{Xi}$ : exportaciones de un cierto país en el sector (industria) $\mathrm{i}$;

$\mathrm{Mi}$ : importaciones de un cierto pais en el sector (industria) $\mathrm{i}$.

El valor oscila entre cero (especialización intra- industrial completa) y uno (especialización inter-industrial completa). Este índice, referido a una industria (i) específica, permite obtener por agregación la medida del grado de especialización intraindustrial de un pais:

$$
æ=1 / n * \sum_{i=1}^{n} x_{i}
$$




$$
æ=1 / n * \sum_{i=1}^{n} x_{i}
$$

1.3. Indice de Grubel y LLoyd

1.3.1. No ajustado (13) :

$$
\begin{aligned}
& B i j=\frac{(X i j+M i j)-|X i j-M i j|}{(X i j+M i j)} \times 100 \\
& B i j=1-\left[\frac{\left|X_{i j}-M_{i j}\right|}{X i j+M i j}\right] \times 100
\end{aligned}
$$

donde

$$
\begin{aligned}
& \mathrm{i} \text { : industria } \\
& \mathrm{j} \text { : país }
\end{aligned}
$$

Su expresión equivale a la unidad menos el índice de Balassa. Por lo tanto, el valor nulo indica especialización interindustrial y la unidad especialización intra-industrial completa, respectivamente.

Este índice cuantifica la importancia relativa de las exportaciones de productos del sector " $\mathrm{i}$ " por parte del país "j", compensadas por importaciones de productos del mismo sector, como porcentaje del comercio total del sector "i" en el país $\mathrm{j}$.

1.3.2. Ponderado: Grubel y Lloyd propusieron -para cualquier nivel de agregación- un promedio ponderado de los valores $\mathrm{Bij}$; las ponderaciones corresponden a la participación de cada industria en el comercio total del país "j":

$$
B j=\sum B i j \frac{(X i j+M i j)}{i}
$$




$$
B j=\frac{\sum_{i}(X i j+M i j)-\sum|X i j-M i j|}{i} \times 100
$$

1.3.3. Ajustado por desequilibrio comercial: Grubel y Lloyd (14) señalan que $\mathrm{Bj}$ es un índice sesgado a la baja siempre que el comercio total del país "j" sea desequilibrado, o en caso que el promedio lo sea de algún subconjunto de todas las industrias en las cuales las exportaciones e importaciones difieren.

El índice ajustado $(\mathrm{Cj})$ por desequilibrio comercial es:

$$
\begin{aligned}
& \Sigma(\mathrm{Xij}+\mathrm{Mij})-\Sigma|\mathrm{Xij}-\mathrm{Mij}| \\
& \mathrm{i} \quad \mathrm{i} \\
& \mathrm{Cj}=\longrightarrow \times 100
\end{aligned}
$$

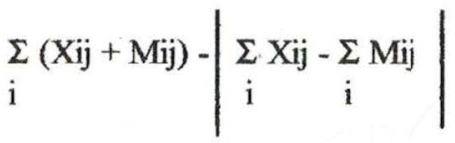

$$
\begin{aligned}
& \mathrm{Bj} \\
& \mathrm{Cj}= \\
& (1-k)
\end{aligned}
$$

donde $\mathrm{k}$ : desequilibrio global del comercio de "j" como proporción del comercio total del país "j".

$$
k=\frac{\left|\begin{array}{c}
\Sigma X_{i j}-\Sigma \text { Mij } \\
i
\end{array}\right|}{\sum_{i}^{\Sigma}\left(X_{i j}+M_{i j}\right)}
$$

El índice se ajusta por sustracción del desequilibrio comercial global del monto total de comercio en el denominador de $\mathrm{Bj}$. En otras palabras, el comercio intraindustrial se mide ahora con respecto al comercio total equilibrado y no al comercio total. 


\subsubsection{Indice de Aquino:}

Aquino (15) considera que el ajuste propuesto por Grubel y Lloyd no detecta (ni corrige) el sesgo en su origen, i.e. a nivel de cada producto ("industria"). En consecuencia, Aquino supone que el efecto desequilibrante es equiproporcional en todas las industrias y equivalente al desequilibrio global.

La corrección del desequilibrio global requiere una estimación de los valores de exportaciones e importaciones de cada bien en condiciones de comercio exterior en equilibrio (exportaciones totales iguales a las importaciones totales). Estos valores "teóricos" de exportaciones e importaciones son:

$$
\begin{aligned}
& \mathrm{X}_{\mathrm{ij}}=\mathrm{Xij} \cdot \frac{1}{2} \cdot \frac{\sum_{\mathrm{i}}(\mathrm{Xij}+\mathrm{Mij})}{\sum_{\mathrm{i}}^{\sum \mathrm{Xij}}} ; \\
& M^{\mathrm{e}_{\mathrm{ij}}}=\mathrm{Mij}_{\mathrm{ij}} \cdot \frac{1}{2} \cdot \frac{\sum(\mathrm{Xij}+\mathrm{Mij})}{\sum_{\mathrm{i}} \mathrm{Mij}}
\end{aligned}
$$

Se verifica que:

$$
\sum_{i} X^{e_{i j}}=\sum_{i} M^{e_{i j}}=\frac{1}{2} \cdot \underset{i}{\Sigma}(X i j+M i j)
$$

La aplicación del índice de Grubel y Lloyd a los valores de $\mathrm{X}^{\mathrm{e}_{\mathrm{ij}}} \mathrm{y}$ $\mathrm{M}^{\mathrm{e}}$ ij permite obtener una medida de la proporción del comercio intraindustrial en el comercio del país " $\mathrm{j}$ " respecto del bien " $\mathrm{i}$ ", "depurado" del efecto resultante del desequilibrio global en el comercio del país " $j "$. Este índice se denomina Qij. El promedio ponderado de los valores de Qij relativos a los distintos bienes (las ponderaciones están dadas por la participación de cada bien en el comercio total de " $\mathrm{j}$ "), es un índice (Qj) corregido de la proporción del comercio intraindustrial sobre el comercio total de "j". 


$$
Q j=\frac{\sum_{i}\left(X_{i j}+M_{i j}\right)-\sum_{i}\left|X e_{i j}-M^{e_{i j}}\right|}{\sum_{i}(X i j+M i j)} \cdot 100
$$

ya que:

$$
\sum_{i}\left(X^{e_{i j}}+M^{e_{i j}}\right)=\sum_{i}^{\Sigma}(X i j+M i j)
$$

Este índice toma valor igual a la unidad cuando todas las industrias consideradas tienen iguales ponderaciones en las exportaciones e importaciones totales; en este caso:

$$
\frac{X_{i j}}{\Sigma X i j}-\frac{M i j}{\Sigma M i j}=0, \text { para todo "i". }
$$

Su valor es mínimo (cero), cuando las importaciones y exportaciones están concentradas en determinadas industrias.

El índice de Aquino, originalmente sugerido por Michaely (16), se basa en el grado de semejanza de la estructura de exportaciones e importaciones de un país. Cuanto más similares son las participaciones de exportaciones e importaciones totales de los productos individuales, mayor será la participación del comercio intraindustrial.

\section{Factores determinantes del comercio intraindustrial (17)}

\subsection{Diferenciación del producto}

El comercio intraindustrial está basado en la diferenciación horizontal y vertical del producto. La primera se refiere a diferencias en las características del producto, relacionadas con combinaciones alternativas de un número dado de atributos (estilo); la segunda indica diferencias de calidad. La industria local puede producir a costos unitarios menores en un rango de calidades, quedando reservado al país extranjero hacerlo en otro segmento, 
según ilustra el siguiente gráfico (18):

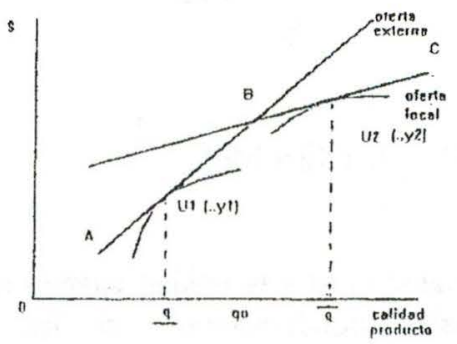

La calidad del producto qo particiona la función de oferta en una fracción de importación (AB) y otra local (BC). Los consumidores de ambos países están ubicados según su función de utilidad (Ui), la cual depende a su vez del ingreso (Yi), sobre la función de oferta $A B C$. Si la distribución del ingreso entre las economias no difiere significativamente, entonces se verifica comercio intraindustrial, tal que los consumidores acceden a variedades de bienes no producidos localmente y el bienestar económico de algunos consumidores en ambos países puede incrementarse.

Tal como sostiene Linder (19), dado que las estructuras nacionales de producción no se diferencian en la misma medida que las de demanda, el comercio intraindustrial crece condicionado por la distribución e intensidad de las preferencias: cuanto más igualitariamente se distribuyan éstas a través de un rango dado de productos, mayor será el potencial para el comercio intraindustrial. Asimismo, a medida que aumenta el ingreso real, aumenta la demanda de "variedad", i.e. la oferta de variedades importadas y los productores locales se disputan los mercados que atraen a los consumidores (nacionales y extranjeros).

\subsection{Economías de escala}

Este factor, relacionado con la diferenciación del producto, responde tanto al enfoque del tamaño óptimo de planta (20), así como a la duración óptima de los procesos necesarios para la producción de bienes diferenciados dentro de la misma planta. En el primer caso, los paises exportarán bienes para los cuales existe una importante demanda de mercado local, el cual brinda a la empresa las economías de escala que le permiten ofrecer en otros mercados su 
propio producto diferenciado a un precio competitivo con los productos externos (si los precios absolutos y relativos de los factores son similares): En el segundo caso, el concepto de economías de escala se refiere a las disminuciones de costo por racionalización (menos productos y series más largas)(21), produciéndose un uso más intensivo de la planta existente y un mayor volumen de producción por período (vg. la industria del acero de un país podría especializarse en la producción y exportación de ciertos productos de acero, mientras que importa otros).

La ampliación de los mercados asociada a la liberalización del comercio permite ofrecer mayor variedad de bienes de consumo (especialización horizontal) o especializarse en la producción de insumos intermedios (especialización vertical). En el primer caso, la diversidad de productos se limita a las empresas nacionales y provoca reducciones de costo a través de la prolongación de las líneas de producción. La especialización vertical se manifiesta en el intercambio de partes y componentes, posibilitando la explotación de economías de escala en la fabricación de dichos insumos.

\subsection{Producción conjunta}

En el caso de coproductos, elaborados en proporciones fijas según la tecnología disponible (vg. destilación de petróleo), el comercio intraindustrial obedece a la compensación de desequilibrios de oferta y demanda.

Si existen varias tecnologías disponibles para producir un bien "A" y los productores en un país (1) disponen de ventajas comparativas en la producción de " $\mathrm{A}$ " mediante tecnologías no-conjuntas, simultáneamente en otro pais (2) se puede producir eficientemente "A" como coproducto en el proceso de fabricación rentable de sustanciales cantidades de "B". En tal caso, el segundo país puede ser también productor y exportador de " $\mathrm{A}$ ".

\subsection{Procesos ahorradores de mano de obra}

El objeto es complementar procesos de producción intensivos en mano de obra y reexportar el producto final al pais de origen de la producción, comportamiento típico de empresas multinacionales (tareas de ensamblaje) que contratan bajos salarios vía la exportación de productos intermedios hacia otro país. 


\subsection{Ciclo del producto}

El comercio intraindustrial se asocia también a la introducción de productos (o procesos) nuevos en un país, los cuales gozan de una protección natural en la primera fase del ciclo de vida y, por consiguiente, no se pueden producir en otro país hasta tanto la tecnología (i.e. el "know how") se haya difundido. Esto se observa especialmente en industrias sujetas a tecnologías en evolución (vg. industria química, farmacéutica, aparatos eléctricos y electrónicos). La extensión del comercio intraindustrial tiende a ser mayor en industrias cuyos productos están en la etapa madura del ciclo de vida y tienen, además, una tasa elevada de desarrollo de variedades del producto (22).

\subsection{Comercio fronterizo}

Es el caso de industrias con costos de transporte elevados respecto a las economías de escala. Puede verificarse incluso en el caso de productos homogéneos o idénticos (cereales, minerales, etc), pero en general se limita a bienes perecederos (alimentos), materiales de construcción y electricidad.

\section{Análisis Empírico}

\section{II.1. Creación y desvío de comercio}

La interpretación de la tendencia de los flujos comerciales se realiza en base a la evolución de la participación de la producción doméstica, las importaciones intra-Mercosur y las importaciones procedentes de terceros países en el consumo doméstico de los países miembro del Mercosur, en base a la metodología sugerida por Jacquemin y Sapir (23) en el caso de la Comunidad Europea.

En el cálculo de esa participación se emplearon series de producto bruto interno total a precios constantes de mercado para los cuatro países del Mercosur (24).

Los valores de exportaciones, importaciones intra-Mercosur e importaciones desde terceros paises, se obtuvieron de las matrices de comercio intrarregional publicadas en el Anuario Estadístico para América Latina y el 
Caribe. Estos valores fueron deflactados por el índice de valor unitario de las exportaciones correspondiente a cada país.

El consumo aparente de cada país miembro del Mercosur puede expresarse como:

$$
\mathrm{C}=(\mathrm{P}-\mathrm{X})+\mathrm{M}^{\mathrm{I}}+\mathrm{M}^{\mathrm{X}} \text {, donde: }
$$

C = consumo aparente

$\mathrm{P}-\mathrm{X}=$ producción doméstica neta de exportaciones

$\mathrm{M}^{\mathrm{I}} \quad=$ importaciones intra-Mercosur

$\mathrm{M}^{\mathrm{X}}=$ importaciones procedentes de terceros países.

Los cambios en las tres componentes del consumo aparente pueden corresponder a una de las siguientes configuraciones:

\begin{tabular}{|l|c|c|c|}
\hline \multirow{2}{*}{ CASOS } & \multicolumn{3}{|c|}{ Cambio en la participación } \\
\cline { 2 - 4 } & $(\mathrm{P}-\mathrm{X})$ & $\mathrm{M}^{\mathrm{I}}$ & $\mathrm{M}^{\mathrm{X}}$ \\
\hline 1 & - & + & + \\
2 & - & - & + \\
3 & - & + & - \\
4 & + & + & - \\
5 & + & - & - \\
6 & + & - & + \\
\hline
\end{tabular}

Los casos 1 y 3 implican Creación de Comercio, caracterizada por la creciente dependencia de los países del Mercosur respecto de sus socios en esa agrupación, como resultado del aumento de la cuota de importaciones procedente desde los mismos.

Los casos 3, 4 y 5 implican Desvío de Comercio, el cual refleja un aumento de la participación de la producción doméstica y/o de las importaciones del Mercosur, a expensas de aquella procedente de importaciones desde terceros países. 
No obstante, cabe observar la dificultad existente en cuanto a la consideración de otros factores que pueden influir asimismo sobre el patrón de comercio, tales como los ajustes en el tipo de cambio, entre otros.

El Cuadro $\mathrm{N}^{\circ} 1$ ilustra los resultados obtenidos según la metodología descripta.

CUADRO $N^{\circ}$ 1: Participación de la producción doméstica $(\mathrm{P}-\mathrm{X})$, importaciones intra-Mercosur $\left(\mathrm{M}^{\mathrm{I}}\right)$ e importaciones extra-Mercosur $\left(\mathrm{M}^{\mathrm{X}}\right)$ en el consumo aparente $(\%)$

\begin{tabular}{|l|l|l|l|l|l|l|l|l|l|l|l|l|l|l|}
\hline Años & \multicolumn{2}{|c|}{ ARGENTINA } & \multicolumn{3}{|c|}{ BRASIL } & \multicolumn{3}{|c|}{ PARAGUAY } & \multicolumn{3}{|c|}{ URUGUAY } \\
\hline & P-X & $\mathrm{M}^{\mathrm{I}}$ & $\mathrm{M}^{\mathrm{X}}$ & $\mathrm{P}-\mathrm{X}$ & $\mathrm{M}^{\mathrm{I}}$ & $\mathrm{M}^{\mathrm{X}}$ & $\mathrm{P}-\mathrm{X}$ & $\mathrm{M}^{\mathrm{I}}$ & $\mathrm{M}^{\mathrm{X}}$ & $\mathrm{P}-\mathrm{X}$ & $\mathrm{M}^{\mathrm{I}}$ & $\mathrm{M}^{\mathrm{X}}$ \\
& & & & & & & & & & & & \\
\hline 1970 & 0.93 & 0.01 & 0.06 & 0.89 & 0.01 & 0.10 & 0.85 & 0.06 & 0.10 & 0.78 & 0.06 & 0.17 \\
1980 & 0.91 & 0.01 & 0.08 & 0.90 & 0.00 & 0.09 & 0.78 & 0.13 & 0.09 & 0.77 & 0.07 & 0.16 \\
1982 & 0.94 & 0.01 & 0.05 & 0.92 & 0.00 & 0.07 & 0.84 & 0.10 & 0.06 & 0.82 & 0.04 & 0.13 \\
1985 & 0.96 & 0.01 & 0.03 & 0.94 & 0.00 & 0.06 & 0.81 & 0.11 & 0.08 & 0.84 & 0.05 & 0.11 \\
1986 & 0.95 & 0.01 & 0.04 & 0.93 & 0.01 & 0.06 & 0.76 & 0.13 & 0.11 & 0.81 & 0.09 & 0.09 \\
1987 & 0.95 & 0.01 & 0.04 & 0.93 & 0.00 & 0.06 & 0.77 & 0.10 & 0.13 & 0.80 & 0.10 & 0.10 \\
1988 & 0.95 & 0.01 & 0.04 & 0.93 & 0.00 & 0.06 & 0.77 & 0.09 & 0.14 & 0.79 & 0.12 & 0.09 \\
1989 & 0.96 & 0.01 & 0.03 & 0.93 & 0.01 & 0.06 & 0.76 & 0.12 & 0.11 & 0.79 & 0.12 & 0.09 \\
1990 & 0.96 & 0.01 & 0.03 & 0.92 & 0.01 & 0.06 & 0.66 & 0.11 & 0.23 & 0.79 & 0.09 & 0.12 \\
1991 & 0.94 & 0.01 & 0.05 & 0.92 & 0.01 & 0.07 & 0.66 & 0.12 & 0.21 & 0.76 & 0.09 & 0.14 \\
1992 & 0.92 & 0.02 & 0.06 & 0.92 & 0.01 & 0.07 & 0.67 & 0.15 & 0.18 & 0.74 & 0.11 & 0.14 \\
\hline
\end{tabular}

FUENTE: Elaboración propia

* Argentina: En los años 1991 y 1992 se observa una caída de la participación de la producción doméstica en el consumo aparente. Las importaciones desde los restantes países del Mercosur tienen una participación constante hasta el año 1991 y en 1992 indica creación de comercio.

Se observa asimismo un aumento de la participación de las importaciones procedentes del resto del mundo. Durante los años 1991/92 se destaca el mayor grado de apertura comercial alcanzado, ya que la 
participación conjunta de las importaciones procedentes del Mercosur y de terceros países pasa de 4\% en 1990 a un 8\% en 1992.

* Brasil: Se observa una caída de la participación de la producción doméstica en el consumo aparente y a partir de 1989 aumenta la participación de las importaciones desde los países del Mercosur, desde un valor casi nulo a un $1 \%$, lo cual denota creación de comercio.

La participación de las importaciones procedentes desde terceros países disminuye desde el año 1985, pasando del 10\% (1970), al 9\% (1980) y finalmente al $6 \%(1985 / 90)$, lo cual indicaría desvío de comercio. Esa participación aumenta a partir de 1991 al $7 \%$, lo cual podría asociarse a una política comercial de mayor apertura seguida por Brasil.

* Paraguay: La participación de las importaciones procedentes de los países del Mercosur aumenta a partir de 1990: 11\% del consumo aparente en 1990; $12 \%$ en $1991 ; 15 \%$ en 1992, indicando así creación de comercio. Simultáneamente disminuye la participación de las importaciones extrarregionales ( $23 \%$ del consumo aparente en $1990 ; 21 \%$ en 1991 y $18 \%$ en 1992), lo cual indicaría desvío de comercio.

La participación de la producción doméstica en el consumo aparente (67\%) es baja comparada con Argentina y Brasil y el grado de apertura de la economia es considerablemente mayor, ya que la participación de las importaciones intra y extrarregionales en el año 1992 asciende al 33\%.

* Uruguay: La participación de la producción doméstica en el consumo aparente de este país, si bien es mayor que en Paraguay (74\%), se encuentra por debajo de las de Argentina y Brasil. Desde 1991 se observa un efecto de creación de comercio - la participación de las importaciones intrarregionales pasa del 9 al 11\%. Asimismo, la participación de las importaciones extrarregionales pasa del 12\% (1990) al 14\% (1991-92).

Este país, al igual que Paraguay, se destaca por el grado de apertura comercial (la participación de importaciones intra y extrarregionales en el consumo aparente en 1992 es del 25\%).

\section{II.2. Criterios alternativos}

La contrastación empírica de criterios alternativos para medir el potencial de una unión aduanera(25) - el caso del Mercosur -se presenta en el 
Cuadro $\mathrm{N}^{\circ}$ 2. A partir de éste, se pueden visualizar aspectos positivos y negativos resultantes de dicha verificación.

Los aspectos positivos corresponden al criterio (3) y, parcialmente, al criterio (4).

En el primer caso, i.e. complementariedad en el comercio de los países miembro de la unión respecto a terceros países, ésta es mayor, en la medida que ese intercambio sea predominantemente de tipo interindustrial, comparado con el comercio intrabloque, de carácter antes bien intraindustrial. En el caso de Argentina se verifica que el comercio con el resto del mundo es más complementario que el realizado con el Mercosur como grupo y con sus países miembros, excepto en el caso del comercio con Paraguay, que es de tipo inter- industrial, i.e. complementario.

El cuarto criterio, referido al comercio de los futuros países miembro previo a la formación de la unión aduanera, no se verifica si se considera la participación de ese intercambio en el comercio total de cada país. En efecto, a partir del análisis de exportaciones e importaciones argentinas por paises (Cuadro $\mathrm{N}^{\circ} 3$ ) puede deducirse que los restantes países del Mercosur no han sido los socios comerciales tradicionales de Argentina.

En 1990 Estados Unidos ocupó el primer lugar como destino de las exportaciones argentinas, siendo desplazado por Brasil en el año 1991.

Asimismo, Estados Unidos también constituyó en los años 1990 y 1991 el primer lugar de procedencia de las importaciones argentinas, seguido por Brasil.

Siguen en orden de importancia: Países Bajos, República Federal de Alemania e Italia, como destino de las exportaciones, y República Federal de Alemania, Bolivia e Italia como origen de las importaciones argentinas, entre otros.

Brasil es el único país socio del Mercosur que ha ocupado posiciones importantes en el intercambio comercial argentino previo a la constitución de esa agrupación integracionista. Uruguay y Paraguay detentan participaciones minoritarias.

No obstante, si se mide el comercio intra-Mercosur respecto al intercambio con el resto de los países de ALADI (Cuadro $\mathrm{N}^{\circ} 4$ ) se observa que en 1991 los países socios en el Mercosur constituyen el primer lugar de procedencia de sus importaciones y destino de sus exportaciones, excepto en un caso (exportaciones de Brasil ). Es decir que, dentro de ALADI, los futuros 
CUADRO $N^{\circ}$ 2: Criterios para establecer la creación de una unión aduanera. El caso del Mercosur

\begin{tabular}{|l|c|}
\hline \multicolumn{1}{|c|}{ CRITERIO } & Se daba en 1991 ? \\
\hline $\begin{array}{l}\text { 1. Nivel tarifario promedio antes de la integración } \\
\text { económica : las importaciones procedentes de los } \\
\text { futuros socios comerciales, han sido gravadas con } \\
\text { aranceles mayores que las procedentes de resto } \\
\text { del mundo ? }\end{array}$ & NO \\
\hline $\begin{array}{l}\text { 2. Rol de las Barreras no Arancelarias. } \\
\text { 3. Antes de la integración económica, puede caracterizarse }\end{array}$ & $\begin{array}{l}\text { SI, desde el } \\
\text { punto de } \\
\text { vista de }\end{array}$ \\
$\begin{array}{l}\text { a las importaciones desde terceros países como significati- } \\
\text { países socios, que el comercio entre los socios que se } \\
\text { integran? }\end{array}$ & $\begin{array}{l}\text { Argentina } \\
\text { (excepto } \\
\text { el comercio } \\
\text { con } \\
\text { Paraguay) }\end{array}$ \\
\hline $\begin{array}{l}\text { f. Participación del comercio liberalizado en } \\
\text { la producción y el consumo mundiales } \\
\text { ciales más importantes desde antes del comienzo del } \\
\text { proyecto de integración de integración? }\end{array}$ & $\begin{array}{l}\text { NO, excepto } \\
\text { Brasil en } \\
\text { algún año, } \\
\text { respecto al } \\
\text { comercio } \\
\text { total. }\end{array}$ \\
\hline
\end{tabular}

FUENTE: Elaboración propia 
CUADRO No 3 : Exportaciones e importaciones argentinas (1990-1991)

\begin{tabular}{|c|c|c|c|c|c|}
\hline \multicolumn{3}{|c|}{$\begin{array}{l}\text { Exportaciones argentinas } \\
\text { (millones de U\$S) }\end{array}$} & \multicolumn{3}{|c|}{$\begin{array}{l}\text { Importaciones argentinas } \\
\text { (millones de U\$S) }\end{array}$} \\
\hline País & 1990 & 1991 & País & 1990 & 1991 \\
\hline EEUU & 1699 & 1244 & EEUU & 876 & 1871 \\
\hline Brasil & 1423 & 1488 & Brasil & 718 & 1526 \\
\hline P. Bajos & 1375 & 1328 & Rep. Fed. & & \\
\hline Rep. Fed. & & & Alemania & 408 & 729 \\
\hline Alemana & 651 & 732 & (1) & & \\
\hline Italia & 523 & 574 & Bolivia & 236 & 248 \\
\hline URSS & 499 & 224 & Italia & 201 & 356 \\
\hline Chile & 462 & 487 & Japón & 144 & 255 \\
\hline Japón & 395 & 454 & Francia & 133 & 393 \\
\hline
\end{tabular}

(1) República Federal de Alemania Unificada a partir del 2.10.1990 FUENTE: Boletín de Comercio Exterior Argentino, Ministerio de Economía, Subsecretaria de Industria y Comercio, No36, enero- setiembre 1990, p.22.

paises socios en el Mercosur tendrian entre sí lazos comerciales más fuertes que con el resto de aquella agrupación regional.

De los restantes países de ALADI, los que mayor relación comercial tienen con el Mercosur son Chile y México, seguidos por Bolivia, con valores de intercambio comparativamente mucho menores.

La contrastaciòn empirica del primer y quinto criterio (Cuadro $\mathrm{N}^{\circ} 2$ ) indica perspectivas desfavorables para la formación del Mercosur.

En el primer caso, además de la menor importancia relativa que en la actualidad tienen los aranceles frente a las barreras no arancelarias como instrumentos proteccionistas, los países socios del Mercosur, en tanto miembros de ALADI, tenían una preferencia comercial respecto a terceros paises (26). 
CUADRO No 4 : Comercio intrarregional. Mercosur y resto de ALADI ( U\$Sx $10^{6}$ )

\begin{tabular}{|c|c|c|c|c|c|c|c|c|}
\hline \multicolumn{4}{|c|}{ Importaciones desde } & \multirow[t]{2}{*}{ PAISES } & \multicolumn{4}{|c|}{ Exportaciones hacia } \\
\hline \multicolumn{2}{|c|}{$\begin{array}{l}\text { Primer área } \\
\text { de origen }\end{array}$} & \multicolumn{2}{|c|}{$\begin{array}{l}\text { Segunda área } \\
\text { de origen }\end{array}$} & & \multicolumn{2}{|c|}{$\begin{array}{c}\text { Primer área } \\
\text { de destino }\end{array}$} & \multicolumn{2}{|c|}{$\begin{array}{l}\text { Segunda áre: } \\
\text { de destino }\end{array}$} \\
\hline Mercosur & 1684 & RA & 748 & Argentina & Mercosur & 1977 & $\mathrm{RA}$ & 1391 \\
\hline Mercosur & 2076 & RA & 1236 & Brasil & RA & 2629 & Mercosur & 2309 \\
\hline Mercosur & 684 & $\mathrm{RA}$ & 70 & Paraguay & Mercosur & 259 & RA & 67 \\
\hline Mercosur & 659 & RA & 94 & Uruguay & Mercosur & 558 & RA & 77 \\
\hline Mercosur & 376 & $\mathrm{RA}$ & 162 & Bolivia & Mercosur & 237 & $\mathrm{RA}$ & 94 \\
\hline RA & 635 & Mercosur & ar 243 & Colombia & $\mathrm{RA}$ & 1014 & Mercosur & 95 \\
\hline Mercosur & 1235 & $\mathrm{RA}$ & 654 & Chile & Mercosur & 795 & RA & 474 \\
\hline $\mathrm{RA}$ & 352 & Mercosur & ir 200 & Ecuador & $\mathrm{RA}$ & 327 & Mercosur & 23 \\
\hline Mercosur & 1023 & $\mathrm{RA}$ & 303 & México & RA & 499 & Mercosur & 426 \\
\hline RA & 731 & Mercosur & ir 442 & Perú & RA & 385 & Mercosur & 157 \\
\hline RA & 700 & Mercosur & II -646 & Venezuela & RA & 743 & Mercosur & 417 \\
\hline
\end{tabular}

FUENTE: Elaboración propia en base a datos del Anuario Estadístico de CEPAL para A. Latina y Caribe; 1992.

No obstante, no se debe omitir el hecho que las negociaciones en el marco de ALADI fuesen por producto y no rebajas lineales y automáticas, como las que caracterizan al actual proceso de integración.

De modo que, si bien no se cumple el primer criterio, de aplicación de mayores aranceles a los países socios que al resto del mundo, en muchos casos los menores aranceles no fueron efectivos en cuanto a la creación de comercio intra- comunitario.

El quinto criterio no se verifica en virtud de la participación prácticamente nula que el comercio liberalizado tiene en la producción y consumo de América Latina ( 0,006 y 0,008 respectivamente) y mundial. Las cifras de producción y consumo de América Latina y el Caribe para el año 1993 indican que el comercio liberalizado asciende a USS 4.979 millones (base 1980), mientras que la producción de América Latina y el Caribe es USS $839.963,4$ millones (base 1980) y el consumo de U\$S 630.756,4 (base 1980) (27). Estas participaciones son naturalmente mucho menores a escala mundial. 


\section{II.3. Comercio intraindustrial}

\section{La información estadística}

Los índices globales se calculan en base a datos de intercambio comercial por secciones de la Nomenclatura del Consejo de Cooperación Aduanera del Comercio Exterior (N.C.C.A.). Se obtienen índices para las secciones: II (Productos del reino vegetal); $\mathrm{II}$ (Grasas y aceites-animales y vegetales- y subproductos); IV (Productos de las industrias alimenticias, bebidas, vinagre, tabaco); VI (Industrias químicas y conexas); XVI (Máquinas y aparatos, material eléctrico) y XVII (Material de transporte).

Este análisis se complementa con un estudio a nivel de capítulos de la misma nomenclatura (28). Los valores del intercambio comercial tomados como base están expresados en miles de dólares, valores FOB y CIF para exportaciones e importaciones, respectivamente.

Los indicadores de comercio intraindustrial calculados son: índice de Grubel y Lloyd no ajustado por desequilibrio comercial; índice de Grubel y Lloyd ajustado por desequilibrio comercial e índice de Aquino.

El período seleccionado corresponde a los años 1986-1992, a fin de incluir los dos acuerdos más recientes de integración económica: el Tratado de Asunción (1991), que define el Mercosur, y su antecedente inmediato, el Programa de Integración y Cooperación Argentino-Brasileño (1986).

\section{Resultados del análisis}

\subsection{Global}

En el Cuadro $\mathrm{N}^{\circ} 5$ se detallan los índices de comercio intraindustrial global -índice de Grubel y Lloyd ajustado y no ajustado por desequilibrio comercial e índice de Aquino - de las secciones N.C.C.A. (I-XXI) para Argentina, con respecto al total mundial, al Mercosur y a sus paises constitutivos ,i.e.: Brasil, Paraguay y Uruguay. 
Se observa que todos los valores del índice no ajustado de Grubel y Lloyd correspondientes al Mercosur son significativamente mayores que aquellos calculados para el total mundial, i.e. el comercio intraindustrial intrabloque es mayor que con el mundo.

Los indices no ajustados de Argentina con Brasil y Uruguay son mayores que con Paraguay en todos los casos, lo cual demuestra una mayor especialización intraindustrial entre Argentina, Brasil y Uruguay, que entre Argentina y Paraguay, el país de menor desarrollo industrial relativo del grupo.

CUADRO No5: ARGENTINA. Indices de GRUBEL y LLOYD no ajustado y ajustado por desequilibrio comercial e indice de AQUINO, para bienes manufacturados por regiones o países (1986-1992)

\begin{tabular}{|c|c|c|c|c|c|c|c|c|c|c|}
\hline \multirow{2}{*}{$\begin{array}{l}\text { AÑO } \\
86\end{array}$} & \multirow{2}{*}{\begin{tabular}{|lll}
\multicolumn{2}{|c}{ MUNDO } \\
Bj & B*j $^{*}$ & Qj \\
35 & 43 & 34
\end{tabular}} & \multicolumn{2}{|c|}{$\begin{array}{c}\text { MERCOSUR } \\
\mathrm{Bj}^{*}{ }^{* j} \mathrm{Qj}\end{array}$} & \multicolumn{3}{|c|}{$\begin{array}{r}\text { BRASIL } \\
\text { Bj B*j Qj }\end{array}$} & \multicolumn{3}{|c|}{$\begin{array}{l}\text { PARAGUAY } \\
\text { Bj B*j Qj }\end{array}$} & $\begin{array}{l}\text { URUGUAY } \\
\text { Bj B*j Qj }\end{array}$ \\
\hline & & 56 & 5955 & 43 & 43 & 43 & 18 & 22 & 18 & $\begin{array}{lll}61 & 73 & 61\end{array}$ \\
\hline 87 & $\begin{array}{lll}31 & 40 & 35\end{array}$ & 60 & 6965 & 45 & 57 & 51 & 17 & 18 & 16 & $\begin{array}{lll}61 & 75 & 62\end{array}$ \\
\hline 88 & $\begin{array}{lll}37 & 50 & 35\end{array}$ & 64 & 7771 & 48 & 65 & 57 & 21 & 22 & 22 & $\begin{array}{lll}66 & 81 & 62\end{array}$ \\
\hline 89 & $\begin{array}{lll}38 & 62 & 38\end{array}$ & 65 & $86 \quad 33$ & 55 & 70 & 51 & 14 & 21 & 18 & $\begin{array}{lll}59 & 91 \quad 59\end{array}$ \\
\hline 90 & $\begin{array}{lll}35 & 70 & 39\end{array}$ & 61 & 9458 & 49 & 72 & 45 & 20 & 46 & 29 & $\begin{array}{lll}58 & 97 & 57\end{array}$ \\
\hline 91 & $41 \quad 5041$ & 59 & 6258 & 47 & 47 & 47 & 28 & 72 & 40 & 617160 \\
\hline 92 & $\begin{array}{lll}35 & 38 & 36\end{array}$ & 45 & 5952 & 36 & 54 & 50 & 25 & 65 & 40 & $\begin{array}{lll}51 & 53 & 51\end{array}$ \\
\hline
\end{tabular}

FUENTE: Elaboración propia en base a datos de INDEC

$\mathrm{El}$ índice de Grubel y Lloyd ajustado por desequilibrio comercial es mayor que el no ajustado $\left(\mathrm{B}^{*} \mathrm{j}>\mathrm{Bj}\right)$ en todos los casos considerados, según lo esperado.

El índice de Aquino es mayor que el de Grubel y Lloyd no ajustado $(\mathrm{Qj}>\mathrm{Bj})$ en la mayoría de los casos, pero menor que el de Grubel y Lloyd ajustado. Es decir que si bien el ajuste de Aquino aumenta el grado de comercio intraindustrial medido, no lo hace de manera tan significativa como el índice ajustado de Grubel y Lloyd, el cual sería sesgado al alza. 
En los casos de desequilibrio comercial poco pronunciado, el ajuste del índice de Grubel y Lloyd no aumenta significativamente el comercio intraindustrial medido: en el comercio con Brasil ( año 1986 ) $\mathrm{Bj}=42.6$ y $\mathrm{B}^{*} \mathrm{j}=42.8$, siendo el desequilibrio comercial de U\$S $6.9 \mathrm{MM}(0.005 \%$ del intercambio total); con Paraguay (año 1987) $\mathrm{Bj}=16.8$ y B ${ }^{*} \mathrm{j}=18.1$, siendo el desequilibrio de USS $-9.4 \mathrm{MM}$, i.e. $0.07 \%$ del intercambio. En los casos de mayor diferencia entre los índices de Grubel y Lloyd ajustado y no ajustado, se produce también un desequilibrio comercial considerable: en el comercio con Uruguay (año 1989): $\mathrm{Bj}=59.1 ; \mathrm{B}^{*} \mathrm{j}=91.2 \mathrm{y} \mathrm{Qj}=59.1$, siendo el desequilibrio comercial de U\$S 104.7 MM (35\% del intercambio total).

En 1992, el desequilibrio comercial Argentina-Mercosur representa el $23 \%$ del intercambio total del país con ese bloque comercial, en tanto en 1991 era sólo el 5\%. En forma correspondiente, el índice de comercio intraindustrial global ajustado ( Cuadro $N^{\circ} 4$ ) en 1992 es de $B{ }^{*} j=59$, i.e. $31 \%$ más elevado que el no-ajustado para igual año $(\mathrm{Bj}=45)$.

Este efecto se observa aún con más intensidad en el caso del intercambio Argentina-Brasil: en 1991, el comercio fue considerablemente equilibrado, con un déficit comercial sólo del $1 \%$ del intercambio total. El indice ajustado $\left(\mathrm{B}^{*} \mathrm{j}=47,4\right)$ es apenas superior al no-ajustado $(\mathrm{Bj}=46,9)$. En contraste, en 1992, cuando el desequilibrio comercial se eleva a $33 \%$ del comercio total, el índice ajustado $\left(\mathrm{B}^{*} \mathrm{j}=54\right)$ es el doble que el no-ajustado $(\mathrm{Bj}=$ 36).

En el comercio Argentina-Uruguay, que en 1992 es más equilibrado que en 1991, se produce el efecto inverso, i.e. la diferencia entre el valor de los indices ajustado y no- ajustado es sólo del 4\%. En los casos de comercio desequilibrado, el índice de Aquino no sería representativo, ya que el comercio intraindustrial no aumenta significativamente al ajustar por desequilibrio comercial.

\subsection{Análisis por secciones}

El Cuadro $\mathrm{N}^{\circ} 6$ presenta índices de comercio intraindustrial por secciones seleccionadas de la N.C.C.A. (índice de Grubel y Lloyd no ajustado). 
* Grasas y aceites (animales y vegetales) y subproductos

Marcada especialización interindustrial; el intercambio es superavitario respecto al mundo y al Mercosur, así como también respecto a Brasil y Uruguay. En relación a Paraguay, el intercambio es deficitario durante el período 1986-1989; tendencia que se revierte en 1990 cuando, simultáneamente, disminuyen las importaciones y aumentan las exportaciones argentinas.

* Productos de las industrias alimenticias, bebidas, vinagre y tabaco

Especialización marcadamente interindustrial (comercio superavitario) respecto al mundo. En cambio, en relación al Mercosur, el comercio es intraindustrial.

CUADRO N ${ }^{\circ}$ 6: Comercio intraindustrial argentino por secciones comerciales y regiones (o paises) $\%$

\begin{tabular}{|c|c|c|c|c|c|c|c|c|}
\hline \multirow[t]{2}{*}{ SECCION } & \multicolumn{3}{|c|}{ MUNDO } & \multirow[b]{2}{*}{1992} & \multicolumn{4}{|c|}{ MERCOSUR } \\
\hline & 1986 & 1988 & 1990 & & 1986 & 1988 & 1990 & 1991 \\
\hline II & 20 & 17 & 8 & 14 & 46 & 66 & 14 & 17 \\
\hline III & 2 & 2 & 1 & 3 & 9 & 12 & 10 & 20 \\
\hline IV & 11 & 4 & 5 & 28 & 99 & 90 & 44 & 95 \\
\hline VI & 45 & 61 & 70 & 50 & 68 & 81 & 99 & 83 \\
\hline $\mathrm{XI}$ & 62 & 27 & 29 & 60 & 45 & 92 & 70 & 53 \\
\hline XVI & 38 & 41 & 67 & 20 & 59 & 83 & 86 & 44 \\
\hline XVII & 86 & 82 & 91 & 30 & 97 & 81 & 91 & 39 \\
\hline
\end{tabular}

FUENTE: Elaboración propia en base a datos de INDEC

En el análisis por países se observa que en los años 1986 y 1987 el comercio con Brasil es intraindustrial (el PICAB se estableció en 1986). El comercio en 1991 fue equilibrado, con $B * j=99$.

Respecto a Paraguay, el comercio en general es superavitario, aunque Argentina importa alrededor del $50 \%$ del valor de sus exportaciones, es decir, hay lugar para el comercio intraindustrial. 
En cuanto a Uruguay, no se observa una tendencia definida, ya que el comercio ha sido superavitario o bien deficitario en distintos períodos. El indice más elevado de comercio intraindustrial es el correspondiente al año $1986(\mathrm{Bi}=81 \%)$.

\section{* Industrias químicas y conexas}

El índice de comercio intraindustrial respecto al mundo es menor de $50 \%$; el valor más elevado se alcanza en $1990(\mathrm{Bi}=70 \%)$. Los indices calculados para el Mercosur son relativamente más elevados, acentuándose esta tendencia a partir del año 1987. Asimismo, a nivel de países, el mayor índice de comercio intraindustrial registrado para todo el período corresponde a Uruguay, seguido de Brasil.

El comercio con Paraguay es superavitario para Argentina y de carácter interindustrial.

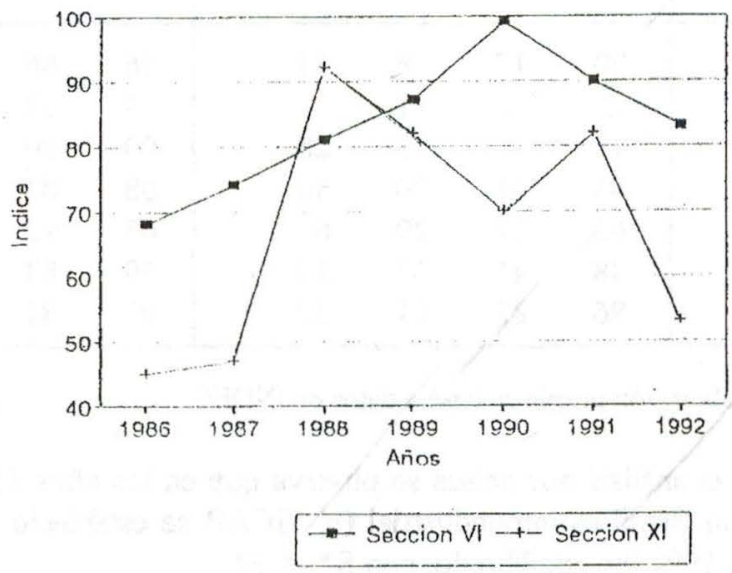




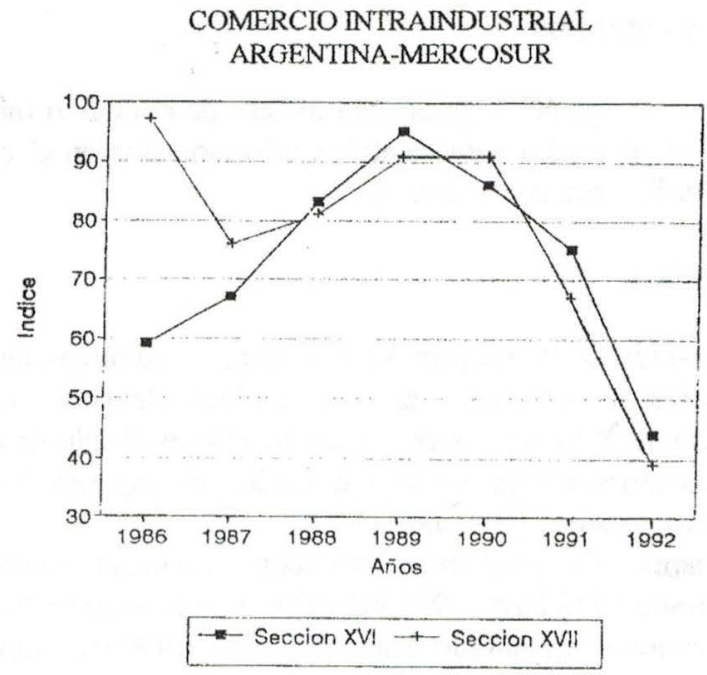

* Máquinas y aparatos, material eléctrico

El comercio en este rubro presenta características de intraindustrial respecto al Mercosur, comparado con el que se realiza con el mundo. Este carácter se acentúa respecto a Brasil a partir de 1989. En cambio, respecto a Paraguay, prevalece un intercambio interindustrial superavitario para Argentina durante todo el período.

En 1991, el comercio con Uruguay refuerza su característica de intraindustrial, con un aumento de las importaciones argentinas en este rubro.

\section{* Material de Transporte}

En general, se observa un índice de comercio intraindustrial elevado respecto al mundo (el valor mínimo de $67 \%$ se produce en 1991), excepto en 1992 , año en que cae a $30 \%$ por la caída generalizada de las exportaciones argentinas.

Los índices respecto al Mercosur son relativamente más elevados, con excepción del año 1988.

A nivel de paises, el mayor índice corresponde a Uruguay (1987), seguido de Brasil (años 1989/90). 


\subsection{Análisis por capítulos}

Los Cuadros $\mathrm{N}^{\circ} 7$ a $\mathrm{N}^{\circ} 9$ presentan índices de comercio intraindustrial (Grubel y Lloyd no ajustado) para capítulos seleccionados en el comercio de Argentina con Brasil, Uruguay y Paraguay.

\section{* Argentina-Brasil}

Los capítulos de la Sección VI ( productos químicos inorgánicos y extractos curtientes o tintòreos) registran índices elevados de comercio intraindustrial durante todo el período, así como valores absolutos elevados de comercio. Un comportamiento semejante exhibe el capítulo 35 (Materias albuminoideas, etc), excepto en el año 1989.

El comercio de productos químicos orgánicos muestra índices representativos desde 1988 hasta 1991 inclusive. Los productos farmacéuticos presentan un comercio equilibrado hacia los años 1990/91, dando lugar a índices elevados de comercio intraindustrial.

CUADRO No 7; Indices de comercio intraindustrial Argentina-Brasil

\begin{tabular}{|c|rrrrrrr|}
\hline Capítulo & 1986 & 1987 & 1988 & 1989 & 1990 & 1991 & 1992 \\
\hline 28 & 70 & 67 & 68 & 54 & 46 & 88 & 4 \\
29 & 21 & 39 & 63 & 62 & 65 & 66 & 3 \\
30 & 1 & 2 & 4 & 52 & 70 & 79 & 1 \\
32 & 92 & 89 & 96 & 57 & 58 & 77 & 5 \\
33 & 19 & 75 & 64 & 51 & 48 & 82 & 1 \\
35 & 76 & 91 & 89 & 43 & 74 & 91 & 11 \\
70 & 88 & 37 & 57 & 35 & 25 & 90 & 6 \\
84 & 57 & 63 & 81 & 92 & 86 & 73 & 5 \\
87 & 86 & 65 & 71 & 93 & 96 & 61 & 2 \\
88 & 0 & 10 & 55 & 84 & 1 & 0 & 0 \\
90 & 22 & 27 & 55 & 62 & 63 & 94 & 5 \\
\hline
\end{tabular}

FUENTE: Elaboración propia en base a datos de INDEC 
El capitulo 33 (aceites esenciales y resinoides) muestra indices elevados desde 1987 a 1991, con excepción del año 1990, cuando se registró un aumento pronunciado de las exportaciones argentinas.

En la Sección XVI, el capítulo 84 (Reactores nucleares, calderas, máquinas, aparatos y artefactos mecánicos, etc.) presenta índices de comercio intraindustrial representativos durante todo el período y valores absolutos de comercio elevados.

En la Sección XVII sobresale el comercio intraindustrial de vehículos automotores, partes, etc, que se mantiene durante todo el periodo, al igual que los elevados valores comercializados. El capítulo 88 ( Navegación aérea o espacial) parece indicar la existencia de comercio intraindustrial durante los años 1988 y 1989 , si bien durante el resto del periodo analizado se producen exportaciones o importaciones netas de Argentina.

COMERCIO INTRAINDUSTRIAL

ARGENTINA-BRASIL

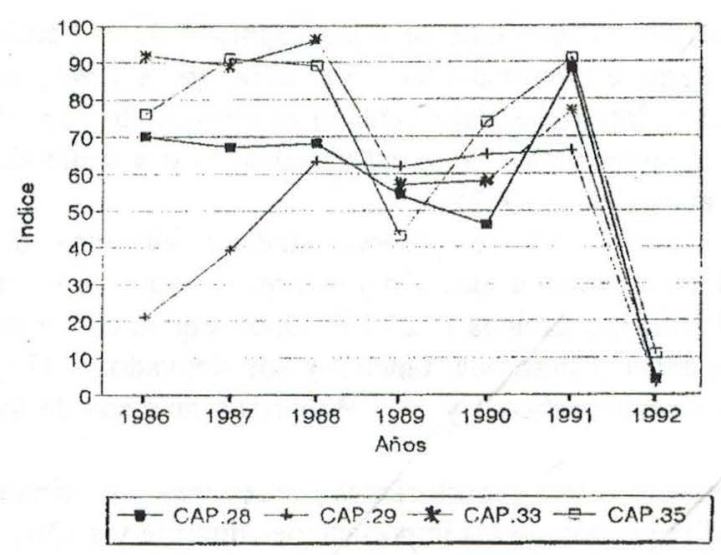


COMERCIO INTRAINDUSTRIAL

ARGENTINA-BRASIL

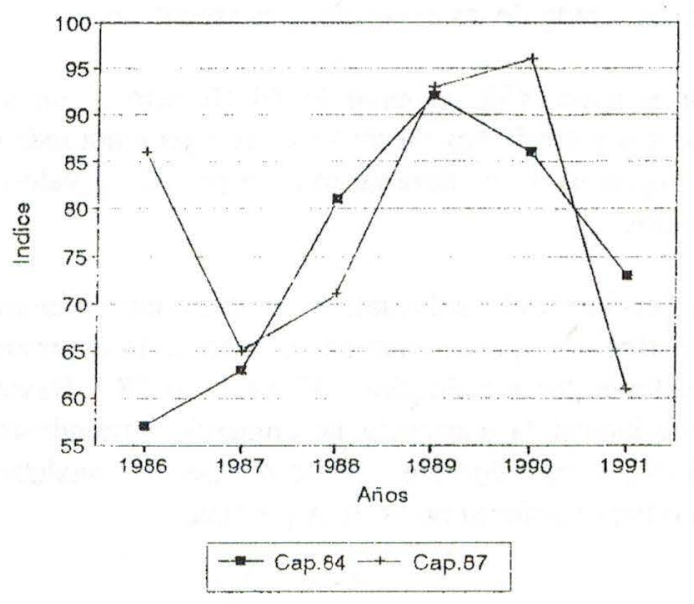

\section{* Argentina - Uruguay}

El comercio correspondiente a los capítulos de la Sección II es en general superavitario e interindustrial. Sin embargo, a nivel agregado, el indice de comercio intraindustrial calculado es elevado, lo cual obedecería a eventuales fluctuaciones de los flujos intercambiados o a comercio fronterizo (cereales en los años 1991 y 1992).

En la Sección VI se observan índices elevados de comercio intraindustrial durante todo el período y valores elevados en los capítulos 28 (Productos químicos inorgánicos) ; 29 (Productos químicos orgánicos ); 32 ( Extractos curtientes o tintòreos; taninos y sus derivados); 37 ( Productos fotográficos o cinematográficos) y 38 (Productos diversos de las industrias químicas).

El aumento de exportaciones argentinas y simultáneamente estabilidad y/o disminución de las importaciones durante los años 1987-1991, permitiría indicar algún grado de comercio intraindustrial en el Capítulo 35 ( Materias albuminoideas). 
PAUTAS PARA EVALUAR EL DESEMPEÑO DE UNA UNION ADUANERA

CUADRO $\mathrm{N}^{\circ}$ 8: Indice de comercio intraindustrial Argentina-Uruguay

\begin{tabular}{|r|rrrrrrr|}
\hline Capitulo & 1986 & 1987 & 1988 & 1989 & 1990 & 1991 & 1992 \\
\hline 7 & 0 & 0 & 2 & 0 & 0 & 12 & 71 \\
8 & 29 & 86 & 66 & 2 & 0 & 49 & 2 \\
9 & 0 & 0 & 1 & 63 & 2 & 16 & 66 \\
10 & 1 & 1 & 2 & 1 & 24 & 72 & 77 \\
11 & 0 & 9 & 21 & 64 & 0 & 11 & 8 \\
12 & 21 & 31 & 29 & 1 & 26 & 39 & 1 \\
28 & 95 & 59 & 81 & 57 & 48 & 83 & 19 \\
29 & 89 & 99 & 94 & 80 & 78 & 80 & 14 \\
32 & 80 & 82 & 67 & 81 & 80 & 65 & 11 \\
35 & 33 & 51 & 54 & 82 & 70 & 66 & 6 \\
37 & 54 & 53 & 71 & 77 & 63 & 77 & 11 \\
38 & 95 & 78 & 99 & 58 & 84 & 95 & 20 \\
39 & 86 & 62 & 66 & 46 & 48 & 63 & 27 \\
40 & 88 & 81 & 39 & 45 & 69 & 32 & 4 \\
51 & 92 & 90 & 99 & 85 & 80 & 99 & 25 \\
55 & 96 & 82 & 63 & 55 & 17 & 74 & 1 \\
85 & 91 & 65 & 66 & 52 & 55 & 85 & 9 \\
87 & 67 & 91 & 76 & 99 & 95 & 83 & 5 \\
89 & 1 & 21 & 91 & 60 & 80 & 4 & 1 \\
90 & 53 & 64 & 76 & 82 & 80 & 77 & 9 \\
91 & 69 & 0 & 53 & 58 & 72 & 76 & 6 \\
92 & 81 & 58 & 94 & 36 & 94 & 2 & 4 \\
\hline
\end{tabular}

FUENTE: Elaboración propia en base a datos de INDEC

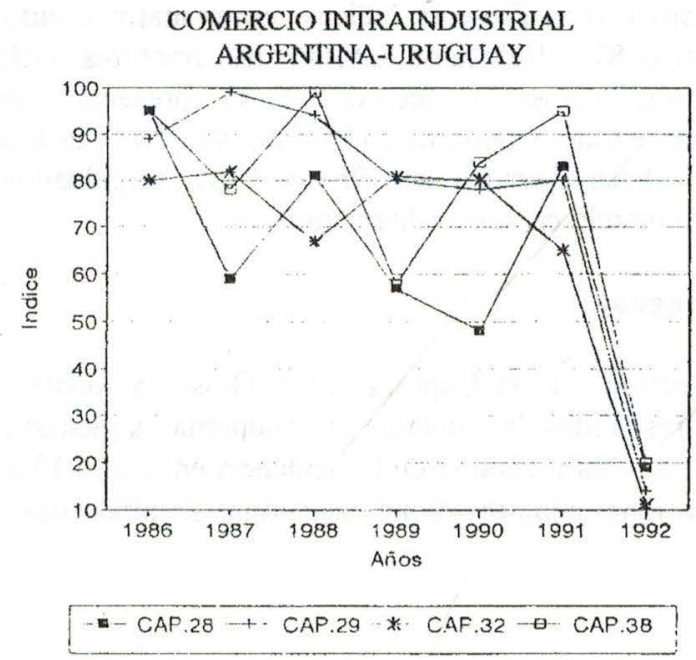




\section{COMERCIO INTRAINDUSTRIAL}

ARGENTINA-URUGUAY

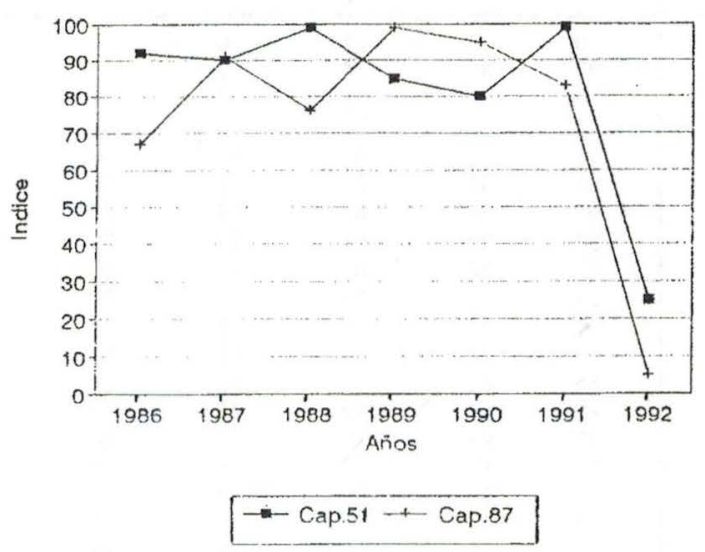

En el Capítulo 51 ( Lana y pelo fino u ordinario, hilados y tejidos de crin) se observan índices de comercio intraindustrial elevados durante todo el período, con flujos de comercio considerablemente equilibrados. Durante los años 1986-89, se destacan los indices de comercio intraindustrial en fibras sintéticas o artificiales discontinuas.

En la Sección XVI, el Capítulo 85 (Máquinas, aparatos y material eléctrico y sus partes, etc.) presenta indices representativos durante todo el período. El Capítulo 87 ( Vehículos automóviles, tractores, ciclos y demás vehículos terrestres, partes y accesorios) se presenta marcadamente intraindustrial durante todo el período. El capítulo 88 ( Navegación marítima o fluvial) presenta índices elevados en 1988 y 1989, respaldados por flujos considerables de comercio en ambas direcciones.

\section{* Argentina-Paraguay}

En la Sección III, el Capítulo 15 ( Grasas y aceites animales o vegetales) arroja resultados de comercio interindustrial, deficitario durante los años 1986-1989, con una reversión de la tendencia en el año 1990, en que se producen exportaciones e importaciones argentinas simultáneamente. 
CUADRO N 9: Indices de comercio intraindustrial Argentina-Paraguay

\begin{tabular}{|l|rrrrrrr|}
\hline Capítulo & 1986 & 1987 & 1988 & 1989 & 1990 & 1991 & 1992 \\
\hline & & & & & & & \\
15 & 2 & 1 & 1 & 2 & 69 & 97 & 20 \\
20 & 52 & 64 & 86 & 65 & 31 & 1 & 7 \\
21 & 46 & 35 & 57 & 66 & 76 & 90 & 31 \\
& & & & & & & \\
\hline
\end{tabular}

FUENTE: Elaboración propia en base a datos de INDEC

En la Sección IV, se analizan los capítulos 20 ( Preparaciones de legumbres $\mathrm{u}$ hortalizas, de frutos o de otras partes de plantas ) y 21 (Preparaciones alimenticias diversas).

En el primer caso, el mayor valor de los índices de comercio intraindustrial corresponde al año $1988(\mathrm{Bi}=86)$. En los restantes años considerados, el comercio es de carácter interindustrial (1990 a 1992) e intraindustrial (1986 a 1989)

En el caso del Capitulo 21 (Preparaciones alimenticias diversas), se observa comercio intraindustrial incipiente $(\mathrm{Bi}=57)$ en el año 1988 y crece sostenidamente hasta el año 1991, en que el índice alcanza un valor $\mathrm{Bi}=90$.

\section{Conclusiones}

1. El índice global de comercio intraindustrial de Grubel y Lloyd ajustado para el comercio de Argentina respecto al mundo y al MERCOSUR, es mayor que el índice no ajustado, diferencia aún más significativa en el caso de existir desequilibrios comerciales.

2. El índice de Aquino es, en general, mayor o igual que el índice no ajustado de Grubel y Lloyd. Este resultado se refuerza si el análisis se desagrega a nivel de países.

3. Los indices globales de comercio intraindustrial Argentina-MERCOSUR son mayores que aquellos referentes al comercio Argentina-mundo. Este resultado se refuerza en el período de comercio administrado 1986-88, durante el cual las diferencias entre el índice de Aquino calculado para el MERCOSUR 
y el mundo son crecientes. Sin embargo, esta tendencia no se mantiene a partir de 1989.

4. Los mayores indices de comercio intraindustrial de Argentina en el MERCOSUR corresponde a las secciones VI (Industrias químicas y conexas); IV (Productos alimenticios) y XVII (Equipo de transporte), en ese orden.

Si se excluye el año 1992, los índices más elevados corresponden a las secciones XVII y VI.

También se observa comercio intraindustrial en las secciones XVI (Maquinaria y equipo, material eléctrico) y XI (Industria textil).

5. El análisis de los flujos comerciales entre Argentina y el MERCOSUR, por países y capítulos, revela la existencia de comercio intraindustrial, aunque sin coincidir en todos los casos.

6. Entre Argentina y Brasil, los mayores índices de comercio intraindustrial corresponden a los Capítulos 32 (Extractos curtientes o tintòreos); 35 (Materias albuminoideas) y 87 (Vehículos automóviles, tractores).

En orden de importancia, le siguen los Capitulos 84 (Reactores nucleares, calderas) y 28 (Productos químicos inorgánicos).

7. En el caso de Argentina y Uiuguay, los índices de comercio intraindustrial más elevados se jóservan en los Capítulos 28 (Productos químicos inorgánicos); 29 (Productos químicos orgánicos); 32 (Extractos curtientes o tintóreos); 37 (Productos fotográficos); 38 (Otros productos químicos); 51 (I ana y pelo fino u ordinario); 85 (Máquinas, aparatos y material eléctrico) y 87 (Vehículos y sus partes).

8. Entre Argentina y Paraguay, el mayor índice de comercio intraindustrial se observa sólo en el Capítulo 21 (Preparaciones alimenticias diversas).

Flujos comerciales en ambas direcciones, aunque de carácier interindustrial, también se registran en los Capitulos 20 (Pręãáaciones de legumbres u hortalizas) y 15 (Grasas y aceites animales o vegetales).

9. Los resultados obtenidos a nivel de capítulo confirman, en general, aquellos calculados por secciones comerciales.

10. En 1992 ningún índice de comercio intraindustrial resultó significativo, debido a que el comercio de Argentina en ese año se torna marcadamente 
deficitario.

Este resultado puede estar condicionado por el nivel de agregación del análisis (secciones y capítulos). En consecuencia, correspondería profundizar esta investigación procediendo a un estudio por productos.

11. Antes de la creación del Mercosur, sus países miembros ya estaban sujetos a preferencias comerciales recíprocas en el marco de la Asociación Latinoamericana de Integración. Por lo tanto, no se verifica el primer criterio de buen desempeño de una unión aduanera, i.e. que las importaciones procedentes de los futuros socios comerciales antes de la integración sean gravadas con aranceles mayores que las procedentes del resto del mundo.

12. Se hace el supuesto de la neutralidad de las barreras no arancelarias respecto a terceros paises en virtud de la dificultad de su identificación y cuantificaciòn.

13. El criterio de complementariedad de las importaciones desde terceros países con respecto a la exportación de los países socios, en contraste con el comercio intra- bloque se verifica en el caso de Argentina respecto a Brasil y a Uruguay, ya que en el comercio argentino con estos países predomina el elemento intraindustrial, frente al comercio con el resto del mundo, que es de tipo interindustrial. No obstante, cabe realizar un análisis más desagregado del "resto del mundo", considerando, por ejemplo, el comercio argentino-chileno, dada la proximidad geográfica y las relaciones de intercambio con ese país, que han llevado a preveer su inclusión en el Mercosur.

El comercio argentino-paraguayo, al ser de tipo inter- industrial, no verifica el criterio enunciado.

14. El criterio de la importancia del comercio entre los paises miembros antes de la integración económica se verifica sólo parcialmente en el caso de Brasil, el cual ha desplazado -en ocasiones - a los socios comerciales tradicionales de Argentina. Ese lugar ha sido ocupado alternativamente por Estados Unidos, la Unión Soviética y los Países Bajos, como destino de las exportaciones argentinas, y por esos mismos paises, además de Brasil y Alemania Federal, como origen de las importaciones argentinas. No obstante, si se restringe el ámbito de referencia a la ALADI, los países del Mercosur verifican mayor intercambio comercial intrabloque que con el resto de aquella agrupación subregional. 
15. El criterio de la participación del comercio liberalizado en la producción y el consumo mundiales no se verifica, ya que la misma sería ínfima.

16. Los criterios enunciados no permiten extraer conclusiones a priori acerca del desempeño de la unión aduanera en el caso del Mercosur, ya que sólo se verifican algunos de ellos, y en general parcialmente.

No obstante, cabría analizar el rol de la eliminación de las barreras no arancelarias en la expansión del comercio intrabloque, debido a su importancia creciente como instrumento proteccionista frente a los aranceles, rebajados a través de las sucesivas "rondas" del GATT.

17. Asimismo, se debe asignar la debida importancia a la representatividad del comercio intraindustrial intrabloque comparado con el realizado con terceros países, y al mayor intercambio observado entre paises del Mercosur frente al resto de ALADI, si bien esto último implica restringir el criterio de la importancia del comercio intrabloque respecto al comercio mundial.

18. En Uruguay y Paraguay la participación de la producción doméstica en el consumo aparente es menor que en Argentina y Brasil, lo cual indica que estos países son más dependientes de las importaciones que los socios mayores en el Mercosur. La liberalización comercial, en principio, beneficiaría en mayor medida a los países más pequeños del bloque.

19. Se observa creación de comercio en todos los países miembros del Mercosur, relativamente mayor en términos porcentuales para Argentina y Brasil.

20. Se observa desvío de comercio en Brasil y en Paraguay, si bien en el primer país la participación de importaciones desde terceros paises aumenta en 1991, lo cual podría asociarse a la política comercial aperturista implementada.

Paraguay mantiene la tendencia a la disminución de la participación de las importaciones extrarregionales.

21. En Argentina y Uruguay se incrementa la participación de las importaciones desde terceros países.

22. Uruguay y Paraguay son los países miembro del Mercosur con el mayor grado de apertura. En ambos casos, la participación de las importaciones intra 
y extrarregionales en el consumo aparente en el año 1992 asciende al 33 y $25 \%$, respectivamente.

23. Si bien el valor de la participación de las importaciones intra y extrarregionales en el consumo aparente en Argentina es considerablemente menor que en los casos anteriores, es destacable, durante los años 1991/92, el mayor grado de apertura alcanzado por este país.

\section{Fabiana Inés López Departamento de Economía Universidad Nacional del Sur}

\section{NOTAS}

(1) Existe discriminación geográfica cuando un mismo bien paga aranceles diferentes según su pais de origen.

(2) VINER Jacob: The Customs Union Issue; Camegie Endowment for International Peace, Nueva York, 1950.

(3).: LIPSEY,R.G "Exámen general de la teoría de las umiones aduaneras"; publicado en: CAVES,R. y JOHNSON,H.: Ensayos de Economía Internacional, Amorrortu, Bs. As., 1971.

(4) ZIPPEL, W.:"Zollunionstheorie. Eine Einfuhrung";WIST, Wirtschaftswissenschaftliches Studium, 1980, Ed.Franz Vahlen y C.H.Beck, Munich y Frankfurt, pp.368-372.

(5) ZIIPPEL, W.: op. cit.

(6) VINER,J.: op. cit.

(7) DREZE, Jacques: "Les exportations intra-CEE en 1958 et la position Belge"; Recherches Economiques de Louvain; Vol.27; 1961; pp.717-738; VERDOORN, P.J.: 'The intra- block trade of Benelux"; En: E.A.G.ROBINSON (Ed.), Economic Consequences of the size of nations; Londres, 1960, pp.291-329; BAI.ASSA,B.: Economic development and integration, Mexico, 1965. (8) GRUBEL y LLOYD: "Intra-industry trade: the theory and measurement of international trade in differentiated products"; Journal of International Economics; Vol.6, $\mathrm{N}^{\circ} 3$, agosto 1976.

(9) ETHIER,W.: "Internationally decreasing costs and world trade"; Journal of International Economics, $\mathrm{N}^{\circ} 9$, febrero 1979, pp.1-24; "National and international returns to scale in the 


\section{ESTUDIOS ECONOMICOS}

modern theory of international trade", American Economic Review; 72, junio 1982, pp.389- 405; KRUGMAN, P.: "New theories of trade among industrial countries", American Economic Review, Papers and proceedings, 73, mayo 1983, pp.343-347; BRANDER,J.: "Intra-industrial trade in identical commodities", Journal of International Economics, 11, febrero 1981, pp.1-14; FALVEY,R.: "Commercial policy and intra-industry trade", Journal of International Economics, Vol.11, 1981, pp.495-511; HELPMAN,E.: "International trade in the presence of product differentiation, economies of scale and monopolistic competition: a Chamberlin-Heckscher-Ohlin approach", Journal of International Economics, 11, agosto 1981, pp.305-340.

(10) BALASSA, B.: "El comercio intraindustrial y la integración de los paises en desarrollo dentro de la economia mundial"; Integración Latinoamericana, No 37, julio 1979,pp.3-16.

(11) FREUND, John E.:"Modern Elementary Statistics"; Prentice-Hall, Englewood Cliffs, N.J., 1967, pp.364-366.

(12) BAL.ASSA, B.: op. cit.

(13) GRUBEL,H.G.; LLOYD,P.J.: op. cit.

(14) GRUBEL, Herbert G. y LLOYD, P.J.: op.cit. "Con un desequilibrio entre exportaciones e importaciones, el promedio debe ser menor que 100, independientemente del patrón de exportaciones e importaciones, porque las exportaciones no pueden igualar a las importaciones en todas las industrias. Esto se debe a que el indice capta tanto el desequilibrio comercial como la intensidad del comercio intraindustrial".

(15) AQUINO, A.: "Intra-industry trade and inter-industry specialization as concurrent sources of international trade in manufactures"; Weltwirtschafiliches Archiv, 1978, N¹14, Vol.2.

(16) La expresión Qj puede simplificarse para obtener la siguiente fórmula, que de hecho es idéntica a la de un indice empleado por Michaely (1962):

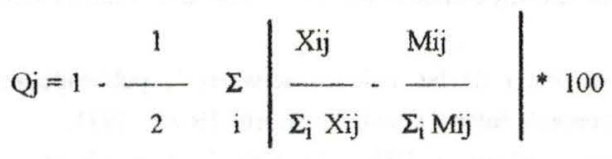

(17) SOURROUILLE y LUCANGELI: "El intercambio comercial argentino-brasileño. Un examen del comercio intraindustrial"; BID-INTAL, Publicación No 389, Buenos Aires, marzo 1992.

(18) BROLL, Udo y GILROY, Michael: "Intraindustrieller Außenhandel"; Wist Heft, No 7, julio 1987,pp.360.

(19) LINDER,S.B.:" An essay on trade and transformation"; Estocolmo, 1961, p. 102.

(20) Idem.

(21) DRABEK y GREENAWAY: "Economic integration and intra- industry trade: the EEC and CMEA compared"; Kyklos; Vol.37, Fasc.3, 1984, pp.444-469.

(22) $\mathrm{TOH}$, Kiertisak: "A cross-section analysis of intraindustry trade in U.S. manufacturing industries"; Weltwirtschaftliches Archiv, Vol.118, N², pp.281-301. 
(23) JACQUEMIN,A. y SAPIR,A: "European Integration or World Integration?"; Weltwirtschaftliches Archiv, Vol.124, No 1; 1988, pp.127-139.

(24) CEPAL: Anuario estadístico para América Latina y el Caribe. 1993

(25) ZIPPEL, W.: op. cit

(26) Asimismo, en la Tercera Reunión del Consejo de Ministros de Relaciones Exteriores de ALADI se amplió el margen de Preferencia Arancelaria Regional: de una franja de 2 a $10 \%$, con un promedio de $5 \%$, se pasó a una de 4 a $20 \%$, con un promedio del $10 \%$-intervalo comprendido entre el $11 \%$ y el $22 \%$ en el caso de los países mediterráneos- (ALADI/CM/Resolución 16 (III), 12.03.87) y se suscribió el Acuerdo Regional para la Recuperación y Expansión del Comercio, con una media del $60 \%$ para la preferencia regional, y un rango entre 40 y $80 \%$ según el grado de desarrollo relativo y $10 \%$ adicional para los paises mediterráneos (ALADI/CM/Resolución 15 (III), 12.03.87).

(27) Anuario Estadistico de CEPAL para América Latina y el Caribe. Año 1993.

(28) Se analizan los capitulos: 6 a $15 ; 20,21 ; 28$ al 32;33; 35; 37 al 40; 51; 55; 70; 84 y $85 ; 87$ al 92.

\section{BIBLIOGRAFIA}

AQUINO,A.: "Intra-industry trade and inter-industry specialization as concurrent sources of international trade in manufactures", Weltwirtschaftliches Archiv 1978 , $\mathrm{N}^{\circ} 114$,Vol.2, pp.275-296.

BALASSA,B.: Economic development and integration, Mexico, 1965.

BALASSA, B.:"El comercio intraindustrial y la integraciòn de los países en desarrollo dentro de la economia mundial"; Integraciòn Latinoamericana, No37, julio 1979 ,pp.3-16.

BANCO PROVINCIA DE BUENOS AIRES: Síntesis, N²98, mayo-junio 1991, p.24.

BHAGWATI, Jagdish: "The pure theory of international trade: a survey"; Economic Journal, marzo 1964, N²93, Vol.LXXIV, pp. 1-84.

BRANDER,J.: "Intra-industrial trade in identical commodities", Journal of International Economics, 11, febrero 1981, pp.1-14.

BROLL,U. y GILROY,M.:"Intraindustrieller Außenhandel", Wist Heft, No7, julio 1987,Ed.F.Vahlen N.C.Beck, Munchen- Frankfurt, pp.360-364.

CEPAL: Anuario Estadístico para América Latina y el Caribe, año 1993.

DRABEK y GREENAWAY: "Economic integration and intra-industry trade: the EEC and CMEA compared"; Kyklos; Vol.37, Fasc.3, 1984, pp.444-469.

DREZE,J.: "Les exportations intra-CEE en 1958 et la position Belge"; Recherches Economiques de Louvain, Vol.27,pp.717-738.

ETHIER,W.: "Internationally decreasing costs and world trade"; Journal of International Economics, $\mathrm{N}^{\circ}$, febrero 1979, pp.1-24. 
ETHIER, W.: "National and international returns to scale in the modern theory of international trade"; American Economic Review;72, junio 1982, pp.389-405.

FALVEY,R: "Commercial policy and intra-industry trade", Journal of International Economics, Vol.11, 1981, pp.495- 511.

FREUND, John E: "Modern elementary Statistics"; Ed. Prentice-Hall, Englewood Cliffs, N.J., 1967, pp.364-366.

GRUBEL y LLOYD: "Intra-industry trade: the theory and measurement of international trade in differentiated products"; Journal of international economics; Vol.6, No3, agosto 1976.

HELPMAN,E: "International trade in the presence of product differentiation, economies of scale and monopolistic competition: a Chamberlin-Heckscher-Ohlin approach",Journal of International Economics, 11, agosto 1981, pp.305-340.

INDEC: Anuario de Comercio Exterior Argentino, varios años.

JACQUEMIN, A. y SAPIR,A.: "European integration or world integration?"; Weltwirtschaftliches Archiv, Vol.124, Nº1, 1988, pp.127-139.

KRUGMAN, P.: "New theories of trade among industrial countries",American Economic Review,Papers and proceedings, 73, mayo 1983, pp.343-347.

LINDER,S.B.: An essay on trade and transformation"; Estocolmo, 1961, p.102.

LIPSEY,R. G.: "Examen general de la teoria de las uniones aduaneras"; Publicado en: CAVES,R. y JOHNSON, H.: Ensayos de Economía Internacional, Amorrortu, Buenos Aires, 1971.

MICHAELY,M:: Concentration in international trade, Contributions to Economic Analysis, 28, Amsterdam, 1962.

SOURROUILLE y LUCANGELI: "El intercambio comercial argentino-brasileño. Un examen del comercio intraindustrial"; BID-INTAL, Publicaciòn N³89, Buenos Aires, marzo 1992.

TOH, Kiertisak: "A cross-section analysis of intraindustry trade in U.S. manufacturing industries"; Weltwirtschaftliches Archiv, Vol.118; 1982, N², pp.281-301.

VERDOORN,P.J.:"The intra-block trade of Benelux", en: ROBINSON, E.A.G.(Ed.), Economic Consequences of the size of nations; Londres, 1960, pp.291-329.

VINER,J.: The Customs Union Issue, Carnegie Endowment for International Peace, Nueva York, 1950.

ZIPPEL,W.:"Zollunionstheorie. Eine Einführung"; WIST, Wirtschaftswissenschaftliches Studium, 1980, Ed. Franz Vahlen y C.H.Beck, Munich y Frankfurt, pp.368-372. 